\title{
MECHANICS AND HISTOLOGY OF SENILE ENTROPION*
}

\author{
BY \\ R. DALGLEISH AND J. L. S. SMITH \\ University Department of Ophthalmology, Manchester Royal Eye Hospital
}

THROUGHOUT recorded history senile entropion of the lower eyelid has contributed to the tribulation of the elderly. The mechanical nature of the condition has prompted a sustained surgical onslaught and at least sixty fairly distinct corrective surgical procedures have been described. Most of these operations have had some modicum of success. This in turn has led to the retrospective creation of aetiological hypotheses or to the amplification of preconceived hypotheses, with little recourse to basic research.

It is the purpose of this paper to present the results of certain investigations in cases of senile entropion of the lower eyelid. Some of these results will be considered in relation to the suggested aetiological hypotheses.

\section{Age and Sex Distribution}

Although the term "senile entropion" is now widely accepted, the appropriateness of the term does not appear to have been tested. During the 5-year period between September, 1958, and September, 1963, 324 patients attended certain out-patient clinics at the Manchester Royal Eye Hospital with this condition. The age distribution of the patients when first attending is shown in Fig. 1. The highest incidence was in the 70-75 year age-group. This distribution tends to support "senile entropion" as a loose, and probably temporary, generic term.

Up to 70 years of age the sex distribution was more or less equal (69 females and 71 males). After 70 years females predominated in the ratio of nearly 2:1 (120 females and 64 males). It is not possible to make accurate deductions from information of this type, but the findings tend to suggest that the incidence is more or less equal in the two sexes.

\section{"Apposition Tension" of the Lower Eyelid}

It will be shown that many authors conceive that lower eyelids which manifest the condition of senile entropion are excessively lax. In general, this estimation is made by the digital distraction of the lid from the underlying globe. It is obvious that the degree of distractibility of the lid incorporates the dual factors of the tension or tone

\footnotetext{
* Received for publication February 19, 1965.
} 


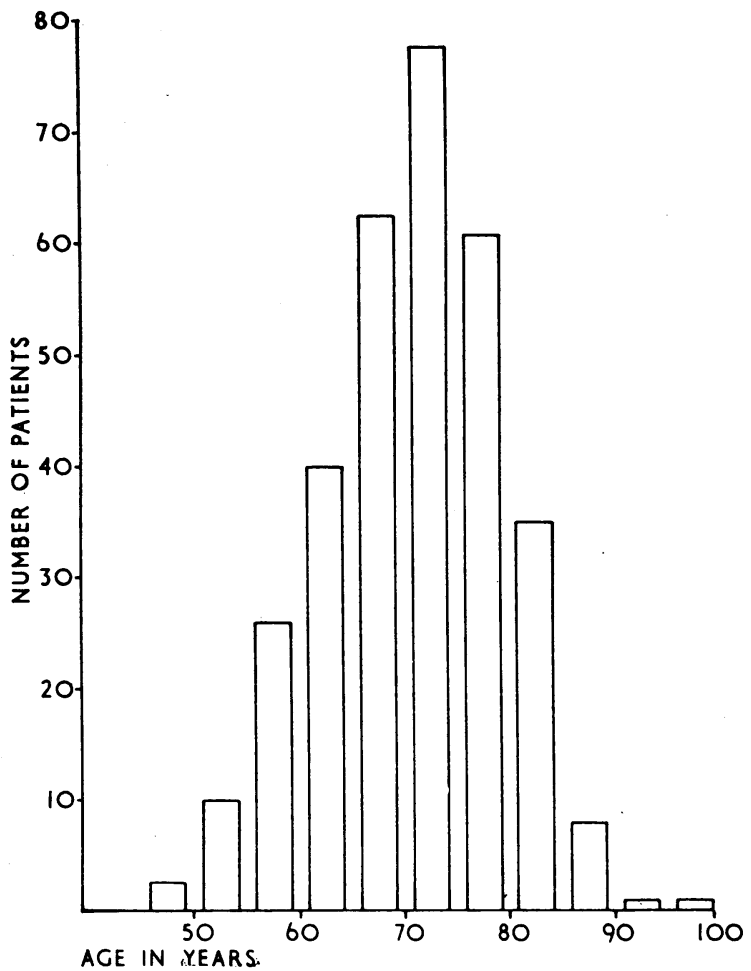

FIG. 1.-Showing age distribution of 324 patients with "senile entropion".

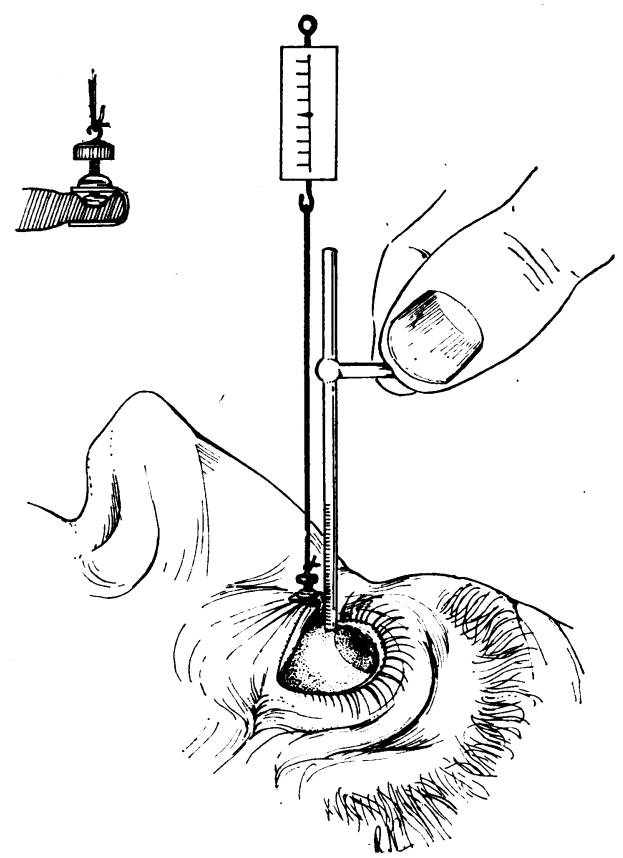

FIG. 2.-Measurement of degree of distraction of inner margin of mid-point of lid from underlying globe. Inset, Modified Castroviejo lid screw-clamp applied to mid-point of lid margin.

of the eyelid itself and the position of the globe (e.g., an enophthalmic eye may give the false impression of a lax.lid). As an expression of the combined effect of these two factors we prefer the term "apposition tension" of the eyelid. This factor has been measured in relation to age in a series of cases of senile entropion and in a control series.

\section{Method}

The patient lies in the supine position with the neck slightly extended. The mid-point of the margin of the lower eyelid is marked with a dot of gentian violet. A tiny subcutaneous bleb of local anaesthetic is raised at that point. Two or three drops of Decicaine are instilled into the conjunctival sac. A Castroviejo lid screw-clamp, modified by the attachment of a small hook to the head of the screw (Fig. 2, inset), is applied to the mid-point of the lid margin. A length of thread connects the lid clamp to an accurate spring-balance which incorporates a mechanism which ensures that it is held vertical to the point of attachment. By varying the vertical position of the spring-balance, traction tensions of $25 \mathrm{~g}$., $50 \mathrm{~g}$., and $75 \mathrm{~g}$. are exerted. Tensions below this order produce inconclusive results because of the weight of the lid itself. The patient is instructed to maintain the eyesin the elevated position. The degree of distraction of the inner margin of the mid-point of the lid from the underlying globe is measured by means of a graduated glass rod, which rests on the globe, and is held adjacent to the lid clamp and parallel with the length of thread (Fig.'2). 
Measurements of this type were taken by an independent observer in a series of 20 cases of senile entropion and in a control series of 36 persons with apparently normal eyelids. The cases were selected to give an even distribution in three age-groups between 50 and 79 years. The result of this investigation is shown in the following Table:

\begin{tabular}{c|c|c|c|c|c|c}
\hline \multirow{2}{*}{$\begin{array}{c}\text { Age Group } \\
\text { (yrs) }\end{array}$} & \multicolumn{5}{|c}{ Mean Distraction (mm.) } \\
\cline { 2 - 7 } & \multicolumn{2}{|c|}{$25 \mathrm{~g}}$. & \multicolumn{2}{|c}{$50 \mathrm{~g}}$. & \multicolumn{2}{c}{$75 \mathrm{~g}}$. \\
\cline { 2 - 7 } & Control & Entropion & Control & Entropion & Control & Entropion \\
\hline $50-59$ & $4 \cdot 1$ & 3.4 & $4 \cdot 8$ & $3 \cdot 7$ & $5 \cdot 3$ & $4 \cdot 1$ \\
\hline $60-69$ & 4.9 & $4 \cdot 8$ & $5 \cdot 8$ & $5 \cdot 0$ & $6 \cdot 4$ & $5 \cdot 3$ \\
\hline $70-79$ & $5 \cdot 7$ & $5 \cdot 5$ & 6.4 & $5 \cdot 7$ & $7 \cdot 5$ & $6 \cdot 1$ \\
\hline
\end{tabular}

With reference to the Table it can be seen that for a given load, the distraction measurement increases with age. Similarly, in each age-group, the distraction measurement increases if the load is increased. These findings apply in the entropion cases and in the controls. However, for any age or load within the limits of this investigation, the distraction measurements in the entropion cases were constantly less than the measurements in the corresponding controls (see Table).

In view of the demonstrated elasticity of the lids (increasing extension under increasing tension), these findings indicate that contrary to popular supposition, the apposition tension of the lower eyelid in cases of entropion is greater than the normal mean for the age-group.

\section{Role of Certain Anatomical Layers of Eyelid in Mechanics of Entropion}

From within outwards, the main anatomical layers of the lower eyelid are as follows:

(1) The conjunctiva.

(2) The tarsal plate, with:

(a) Its well-defined medial and lateral attachments - the palpebral ligaments.

(b) Its ill-defined inferior attachments, which include the expansions from the sheaths of the inferior oblique and inferior rectus muscles, a few smooth muscle fibres, and the orbital septum.

(3) The palpebral part of the orbicularis oculi muscle, which can conveniently be subdivided into:

(a) A marginal portion which lies in the dense tissue adjoining the lid margin.

(b) A pre-tarsal portion which lies anterior to the tarsus.

(c) A pre-septal portion which lies anterior to the orbital septum.

(4) The skin and superficial fascia.

Apart from the general concept of the apposition tension of the full-thickness eyelid, which has been dealt with, it will be shown that all the main anatomical layers 
of the lower eyelid have been implicated, at various times, by various authors, in the multiple aetiological hypotheses relating to senile entropion. Certain investigations have been carried out in an attempt to clarify the position.

\section{Method}

For some years one of us (R.D.) has employed a full-thickness resection of a central segment of the lower eyelid for the correction of senile entropion. The rationale for this operation has been the concept that an eyelid which is in firm apposition with the underlying globe, throughout its full depth, can neither invert nor evert.

The operation (Fig. 3) is carried out under local anaesthesia. The first step is a vertical incision through full-thickness eyelid to one or other side of the midline. The cut edges are then overlapped, using moderate surgical tension. This indicates the width of the segment to be excised. As indicated in Fig. 3, the segment has vertical sides throughout the depth of the tarsal plate, and it tapers to a point below that level. A silk mattress suture unites the lid margin, and interrupted buried chromic catgut sutures unite the tarsal plate and conjunctiva of the lower fornix. In this situation a vertical skin scar is undesirable and it is obviated by means of a Z-plasty.
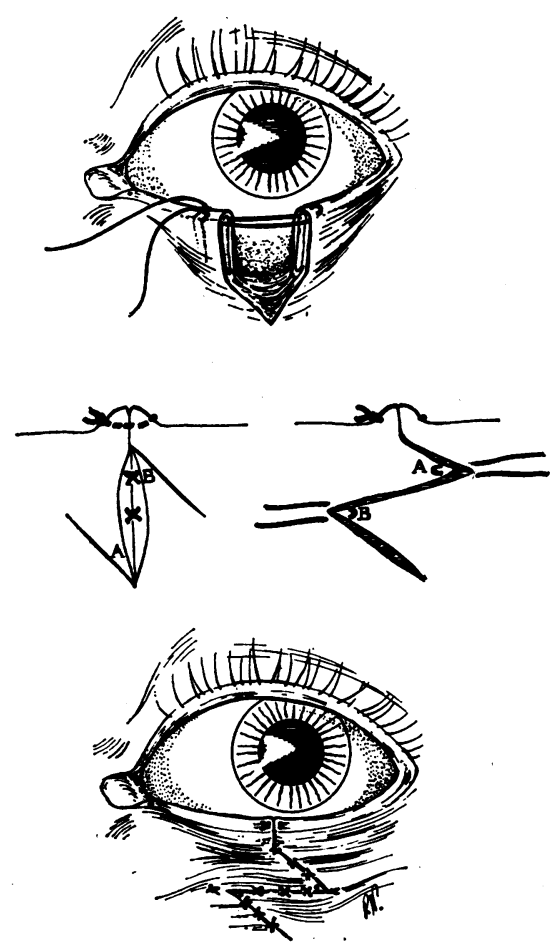
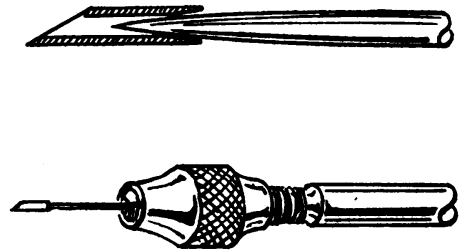

FiG. 4.-Metal marker to be inserted in eyelid. The marker is held by a fine magnetized straight needle.

In certain cases, before carrying out the operation described above, metal markers were inserted in the various main anatomical layers of the lid in the midline (i.e., in the area to be excised). The markers, which were $2-3 \mathrm{~mm}$. pointed segments of 20-gauge hypodermic needle, were delivered into the appropriate anatomical layer on the end of a fine magnetized straight needle (Fig. 4). Lateral radiographs were then taken with: (i) the lower eyelid in the normal position; (ii) the eyelids in a state of forceful closure; (iii) the lower eyelid in the "entropion" position.

Fig. 3.-Showing steps in the operation. Top, Fullthickness segment of lower eyelid excised. Centre and Bottom, Cut edges sutured and Z-plasty fashioned to obviate vertical skin scar. 
Following the excision of the full-thickness segment from the centre of the eyelid, the position of the markers was confirmed by dissection and they were retrieved to be used again.

This type of investigation has been carried out on 8 cases. Enlarged photographic prints were made from the $x$-ray plates and the findings have been analysed. In terms of absolute measurements, the findings have all varied in degree from case to case. However, a constant pattern has emerged which has given a clear indication of the role of certain anatomical layers of the eyelid in the mechanics of entropion. Two cases have been selected to illustrate some of the findings.

Case 1 (Figs. 5, 6, and 7).-Two markers were inserted vertically in the midline of the tarsal plate, one below the other. A third marker is situated horizontally in the pre-tarsal arcades of the orbicularis oculi muscle.

Case 2 (Figs. 8, 9, and 10).-The positioning of the tarsal plate markers was the same as for Case 1. The third and fourth markers are situated horizontally in the pre-septal arcades of the orbicularis oculi muscle.

\section{The Role of the Tarsal Plate}

(1) From the normal position to the position of forceful closure of the eyelids the following change takes place. The upper edge of the tarsal plate rotates inwards, and shifts downwards and backwards. The lower edge of the tarsal plate rotates to a greater degree in the opposite direction and moves forwards with little if any change in its vertical position (compare Fig. 5 with Fig. 6 and Fig. 8 with Fig. 9).

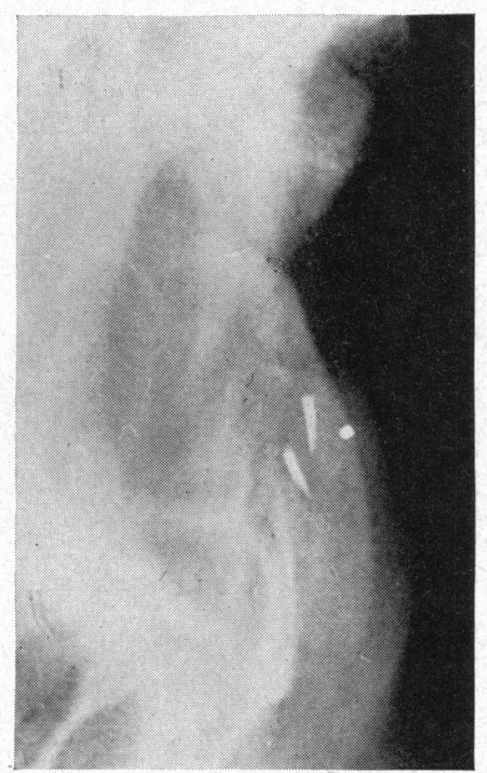

FIG. 5.-Case 1. Lower eyelid in the normal position. The two vertical markers are in the tarsal plate. The horizontal marker is in the pretarsal orbicularis.

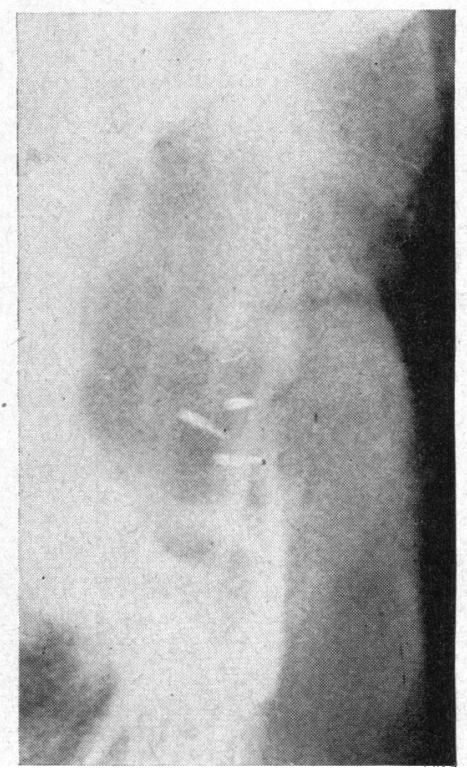

FIG. 6.- Case 1. Eyelids in a state of forceful closure. The upper edge of the tarsal plate has rotated through $59^{\circ}$ and the lower edge through $65^{\circ}$. As compared with Fig. 5 the position of the marker in the pre-tarsal orbicularis is unchanged in relation to the underlying tarsal plate marker. 
(2) From the normal position to the entropion position the following change occurs. The upper edge of the tarsal plate rotates inwards and shifts downwards and backwards. The lower edge of the tarsal plate rotates to a considerably lesser degree in the opposite direction and moves forwards and downwards (compare Fig. 5 with Fig. 7 and Fig. 8 with Fig. 10). The difference in the degree of rotation of the upper and lower edges indicates that the tarsal plate is bent about its long axis in the position of entropion.

\section{The Role of the Pre-tarsal and Pre-septal Arcades of the Orbicularis Oculi Muscle}

(1) The pre-tarsal arcades maintain a fixed relationship with the underlying tarsal plate (compare Figs. 5, 6, and 7). Their movement from the normal position to their position in either forceful closure of the eyelids or entropion is therefore the same as that described for the corresponding portion of the underlying tarsal plate.

(2) The pre-septal arcades, on the other hand, show a considerable change in position in relation to the tarsal plate. In the state of forceful closure of the eyelids these arcades loop forwards and upwards above the level of the tarsal plate to an extent that the vertical inter-relationship of some of the arcades becomes reversed (compare Figs. 8 and 9). In the entropion position, these arcades approximate towards the lower border of the tarsal plate (Fig. 10). This is due to the combined effect of the downward and forward shift of the lower border of the tarsal plate and a slight upward and forward shift of the pre-septal arcades.

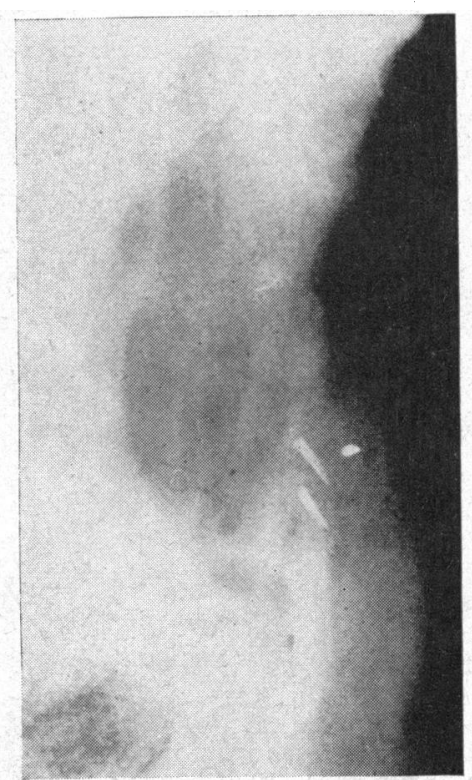

FIG. 7.-Case 1. Lower eyelid in the "entropion" position. From the normal position, the upper edge of the tarsal plate has rotated through $29^{\circ}$ and the lower edge through $6^{\circ}$. The position of the pre-tarsal orbicularis marker is still unchanged in relation to the underlying tarsal plate marker.

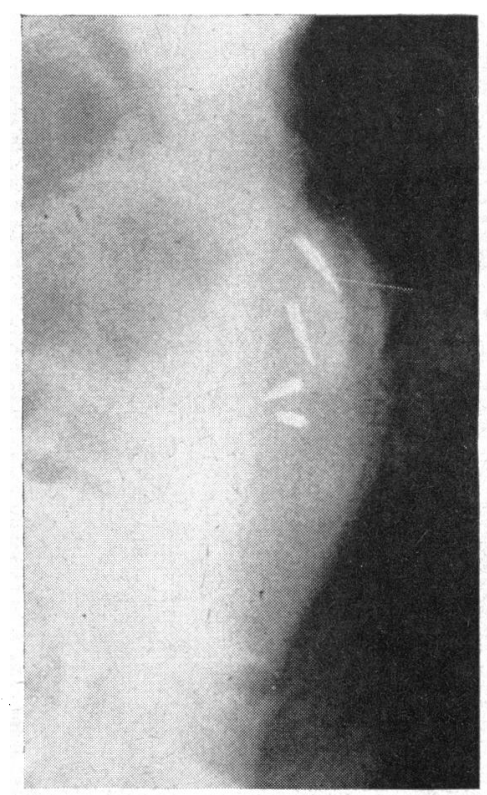

FIG. 8.-Case 2. Lower eyelid in the normal position. The upper two markers (more or less vertically placed) are in the tarsal plate. The lower two markers (horizontally placed) are in the pre-septal orbicularis. 


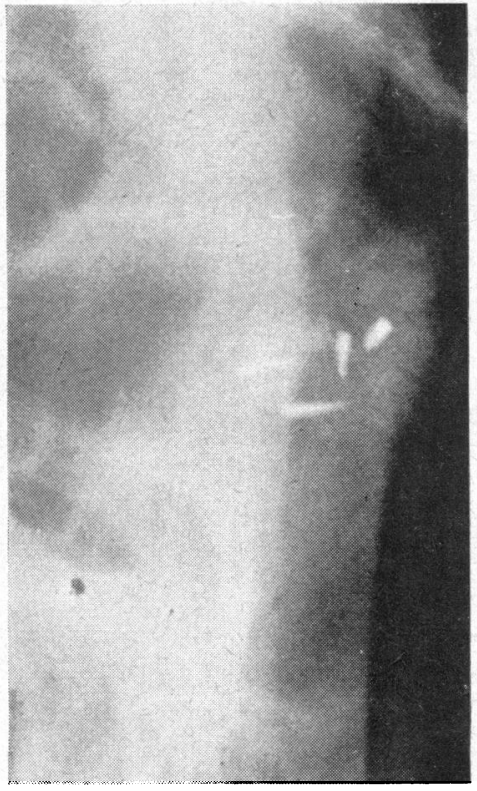

FIG. 9.-Case 2. Eyelids in a state of forceful closure. The upper edge of the tarsal plate has rotated through $60^{\circ}$ and the lower edge through $71^{\circ}$. The pre-septal orbicularis markers are now above the level of the tarsal plate, and the vertical interrelationship of these two markers has been reversed.

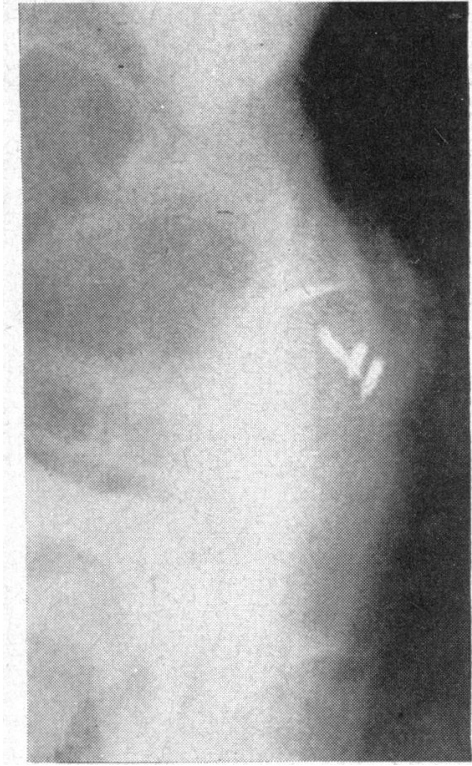

FIG. 10.-Case 2. Lower eyelid in the "entropion" position. From the normal position, the upper edge of the tarsal plate has rotated through $63^{\circ}$ and the lower edge through $16^{\circ}$. The pre-septal orbicularis markers and the lower tarsal plate marker have approximated towards each other.

\section{The Role of the Skin of the Eyelid}

In general terms, the skin of the eyelid would appear to have a passive role in the mechanism of entropion. In both the entropion position and in the state of forceful closure of the eyelids, the position of the lower pre-tarsal and pre-septal skin can be passively changed in relation to the underlying structures. It is therefore not surprising that the vertical, horizontal, and rotational shift of the skin was found to be rather variable, and a detailed presentation would serve no purpose.

\section{Histology}

Twenty-six full-thickness wedge-shaped segments of lower eyelids were subjected to histological examination by one of us (J.L.S.S.). These segments were excised in the surgical correction of senile entropion. As a rule the portions of lid were bisected centrally, the cut being made perpendicular to the lid margin so that each specimen yielded sections at two levels taken from paracentral vertical planes. With three of the specimens the procedure was modified as the length of lid margin measured only $4 \mathrm{~mm}$. These three were sectioned directly, obliquity of the plane of section being prevented by the rectangular shape of the excised tarsal plate. In most cases the structure of the orbital portion of the lid could be amply demonstrated, but in a minority the lower limit of the excision, especially in regard to the deeper and conjunctival surface, was not sufficient for this purpose. 


\section{Structure of the Tarsal Portion of the Eyelids}

In this region the "skeletal" framework is provided by the tarsal plate and the dense collagenous tissue adjoining the lid margin. Enclosed in the latter are three rows of lash follicles which separate incompletely the marginal fibres of the orbicularis from the pre-tarsal component of the muscle. In 16 specimens of this entropion series there was no evidence of distortion in the compact fibrous tissue of the tarsal portions of the lid, the general configuration of which, notably in regard to posterior concavity, direction of lashes, and relatively sharp posterior marginal angle was assessed as normal.

In 7 specimens there was a simple increase in posterior concavity of moderate degree, a process not fully uniform but most emphasized in the central or lower central area of the tarsal plate. In 2 specimens the sectional curvature at the posterior marginal angle appeared sufficiently gentle as to suggest commencing ectropion, and the epithelium to the conjunctival side of the tarsal gland openings, though not abnormally thick for this region, showed a fine granular layer for a distance of some 1 to $2 \mathrm{~mm}$., i.e., a true keratinizing epithelium. The final exception to the general rule of no distortion exhibited an increased posterior concavity of the tarsal plate, together with some flattening of the posterior marginal angle. Here, as over much of the tarsal area of this specimen, the epithelium was of a flattened stratified type and was lacking in the pseudo-glandular invaginations normally seen in this situation. In addition, the conjunctiva differed from that in all the other specimens in being unduly thick and fibrous; this one must relate to antecedent inflammation. In all but these last three specimens the conjunctiva appeared normal in that it showed no undue thickening, remained closely adherent to the tarsal plate throughout its extent, and its epithelium retained a normal character, plentiful goblet cells being found up to the orifices of the Meibomian glands.

In man, the lower tarsal plate forms a band $1 \mathrm{~mm}$. in thickness and $5 \mathrm{~mm}$. in width centrally (Wolff, 1958). In every case the width of the tarsal plate was measured to the nearest $\frac{1}{4}$-millimetre (Fig. 11). In a majority (23 specimens) the mean of two readings is recorded as sections were available from two levels. The commonest measurement obtained was in fact $5 \mathrm{~mm}$., but in four specimens the width of the tarsal plate was in excess of this figure and in 9 cases fell below it. Meibomianitis presented in a few cases, mostly minimal and usually distal, but in 3 specimens it was marked at both levels and had led to some reduction in size of the tarsal plate, the relevant measurements being $4 \mathrm{~mm}$., $4 \mathrm{~mm}$., and $4.5 \mathrm{~mm}$. It was not considered that any significant shrinkage had occurred during processing, as a low-melting-point wax was used.

The tarsal plate is developed as a condensation around the Meibomian glands and its width tends to be slightly reduced outside the immediate gland areas. In the same manner, but more markedly so, the thickness of the tarsal plate is influenced by its glandular content and this is greatest in the lower central area. Measurements in this latter situation gave values mostly between 1.1 and $1.5 \mathrm{~mm}$.; the adjoining pretarsal muscle was, in general, of comparable thickness. All anatomical divisions of the orbicularis muscle seemed fully developed and of normal appearance. 


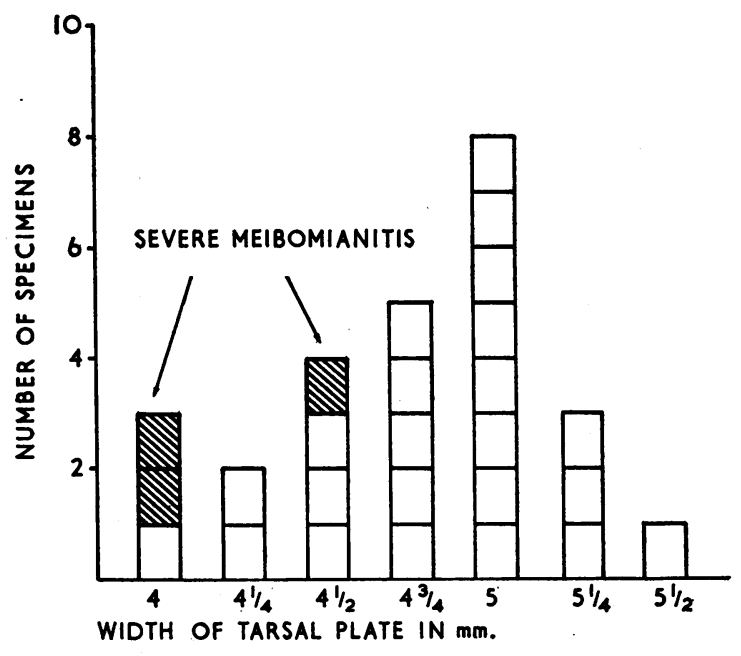

FIG. 11.-Showing measurement of width of tarsal plate to nearest $\frac{1}{4}$-millimetre.

Normally, the dermis immediately beneath the lash line is firmly bound down to the pre-tarsal muscle, but lower down it tends to ride away somewhat with the formation of a potential or actual space bridged by loose connective tissue, in which run small groups of muscle fibres. In the majority of the entropion specimens the dermis appeared more than usually condensed, in the sense of being firmly bound down to the underlying muscle of the entire pre-tarsal area.

\section{Structure of the Orbital Portion of the Eyelids}

Here two main fascial planes are evident, one immediately posterior to the orbicularis fibres-representative of the orbital septum - and one to the deep aspect of the conjunctiva. These two are interconnected, and on occasion this may be so dense for some little distance beneath the tarsal plate as to constitute one layer. Between the two layers lies a variable amount of adipose tissue and some smooth muscle fibres (Muller's muscle).

Six of the specimens could not be assessed owing to the very limited width of tissue excised from this portion of the lid. Of the remainder, 5 showed little fat between the fascial planes and the others moderate or abundant amounts. The structure of the tissues of this region was assessed as being normal in all the specimens.

\section{Discussion}

It has been pointed out that the varying degrees of success of the multiple corrective surgical procedures for senile entropion have led to the creation of many aetiological hypotheses, with little recourse to basic research. The resultant diversity is therefore not surprising and it is indeed difficult to attempt any classification.

Most aetiological hypotheses incorporate several factors which are described as being either causative or accessory in the production of senile entropion. The more 
commonly quoted factors are listed below and some will be examined in the light of our investigations.

(1) Enophthalmos and/or laxity of the full-thickness lower eyelid (Fuchs, 1917; Meek, 1940; Butler, 1948; Fox, 1951; Duke-Elder, 1952; Kirby, 1953; Lebensohn, 1953; Szlazak, 1957; Jones, 1960; Foulds, 1961).

We have defined the term "apposition tension" of the eyelid as an expression of the combined effect of the position of the globe and the tension of the eyelid itself. It has been shown that the apposition tension of the lower eyelid in cases of senile entropion is greater than the normal mean for the age-group. This is conclusive evidence that the lower eyelid itself is certainly not lax. In addition, it is unlikely that there is any disproportionate enophthalmos in relation to age unless this is more than compensated for by an increased tension in the lower eyelid.

(2) Conjunctival inflammation and/or shrinkage (Streatfield, 1858; Duke-Elder, 1952).

With very few exceptions, our histological investigations emphasize the normality of the conjunctiva in these cases.

(3) Atrophy of the tarsal plate (Stallard, 1958), or a small or poorly developed tarsal plate (Adler, 1947).

In 21 out of the 26 histological specimens the width of the tarsal plate was within $\frac{1}{2} \mathrm{~mm}$. of the accepted normal of $5 \mathrm{~mm}$. (Fig. 11). It fell below this limit in 5 cases, two of which were associated with severe meibomianitis, suggesting that any underdevelopment or shrinkage of the tarsal plate is an infrequent and incidental finding in cases of senile entropion.

(4) Stretching of the tarsal plate and medial and lateral palpebral ligaments (Butler, 1948; Lebensohn, 1953; Foulds, 1961).

(5) Stretching or laxity of the orbicularis oculi (Fox, 1951; Szlazak, 1957).

In 20 cases the apposition tension of the lower eyelid has been measured before and then during local akinesia (facial block) or general anaesthesia. The obvious difficulty in obtaining a standardized level of akinesia or general anaesthesia may account for the variability of the results of this investigation. However, it would appear that in the resting state the orbicularis oculi makes a relatively minor contribution to the apposition tension of the lower eyelid. In view of this and the fact that it has been shown that the apposition tension of the lid in cases of entropion is greater than the normal mean, it is extremely unlikely that there is any stretching or laxity of the tarsal plate or palpebral ligaments in cases of entropion. On similar grounds, it is unlikely that there is any stretching or laxity of the orbicularis oculi unless this is more than compensated for by an increased tension in the tarso-ligamentous layer.

(6) Stretching of the inferior attachments of the tarsal plate (Duke-Elder, 1952; Jones, 1960).

Ample evidence of the extreme mobility of the lower part of the tarsal plate in cases of senile entropion has been submitted (Figs 5-10). Although the histological picture is one of normality, it is reasonable to assume that this mobility requires a degree of laxity of the inferior attachments. This laxity may be the result of the normal downward sagging of the lower eyelid which accompanies senility, or it may represent a degree of stretching of these tissues. 
(7) Slackening of the pre-tarsal connective tissue of the lid and upward migration of the pre-tarsal arcades of the orbicularis oculi towards the lid margin (Weeks, 1900; Kettesy, 1948; McFarlane, 1956; Fooks, 1961).

Evidence has been submitted that the pre-tarsal arcades of the orbicularis maintain a fixed relationship with the underlying tarsal plate and that there is no appreciable migration of these arcades towards the lid margin (Figs 5, 6, and 7). The histological sections show that the pre-tarsal arcades have fascial connexions with the tarsal plate with a minimum of intervening areolar tissue.

(8) Loss of attachment of the pre-septal arcades of the orbicularis oculi to the orbital septum, and the upward movement of these arcades between the pre-tarsal muscle and pre-tarsal skin (Jones, 1960).

The upward movement of the pre-septal arcades of the orbicularis in cases of senile entropion is beyond doubt (Figs 8, 9, and 10). However, there is little tendency for these arcades to invade the tarsal portion of the lid as the close adherence of the dermis to the underlying pre-tarsal muscle tends to preclude this. In the state of forceful closure of the eyelids and in the entropion position the pre-septal arcades move upwards and forwards. It is likely that the arcades are pushed forward by the tumbling of the tarsal region of the eyelid, as a forward movement is contrary to their line of action. This must indicate the action of extrinsic mechanical forces on the tarsal region of the eyelid. In this respect we suggest that the vertical interrelationship of the eyelid and the globe may be a factor. In addition, it must be pointed out that most authors have ruled out the possibility of spasm or over-action of the palpebral orbicularis on flimsy or even false deduction. In view of the increased apposition tension of the lower eyelids in these cases it is suggested that this possibility is still open and that further investigation is required in this field.

(9) Atrophy, or loss of tone, or stretching, or excess of skin of the eyelid (Streatfield, 1858; Meek, 1940; Fox, 1951 ; Duke-Elder, 1952; Schimek, 1957; Jones, 1960; Foulds, 1961).

Our histological investigations indicate that these simple "age changes" are in no way emphasized in cases of senile entropion.

It has been pointed out that our clinical investigations indicate that the skin of the lower eyelid would appear to have a passive role in the mechanics of senile entropion.

\section{Surgical Implications}

In a review of the literature relating to the surgical correction of senile entropion of the lower eyelid Schimek (1957) listed more than sixty operations. Since then, at least eight "new" operations have been described.

In view of the multiplicity of the possible mechanical effects of these procedures, it is impractical to attempt to evaluate the implications of our findings in relation to each operation. However, certain generalizations appear justified:

(1) Operations which shorten the tarso-ligamentous layer, on the assumption that this layer is too lax, are based on a false concept.

(2) Operations which aim to prevent "the upward migration of the pre-tarsal arcades of the orbicularis oculi towards the lid margin" are based on a false concept. 
(3) The ageing skin of the lower eyelid has a passive role in the mechanism of senile entropion and operations confined to that layer are generally found to be inadequate.

(4) Judgement should be reserved on operations which aim to reduce the contractile function of the palpebral orbicularis.

(5) At the present time, it would appear rational that surgical intervention should be directed towards the correction of the following:

(a) The forward rotation of the lower edge of the tarsal plate and the buckling of the plate around its long horizontal axis.

(b) The upward and forward movement of the pre-septal arcades of the orbicularis oculi.

\section{Summary}

Evidence is presented which tends to support the acceptance of "senile entropion" as a loose and probably temporary generic term. It is suggested that the incidence of the condition is more or less equal in the two sexes.

The term "apposition tension" of the eyelid is defined. It is shown that in cases of senile entropion the apposition tension of the lower eyelid is greater than the normal mean for the age-group.

By the insertion of metal markers, followed by radiological studies, detailed information has been presented concerning the role of the main anatomical layers of the lid in the mechanics of senile entropion. The forward rotation of the lower edge of the tarsal plate, a horizontal buckling of the plate, and the upward and forward movement of the pre-septal arcades of the orbicularis oculi are notable features. It is suggested that the skin of the eyelid has a passive role in this mechanism.

The findings of the histological examination of 26 full-thickness wedge-shaped segments of lower eyelids are presented. These segments were excised in the surgical correction of senile entropion. Apart from curvature changes in the tarsal plate in a minority of cases, the over-all histological picture is one of normality.

It is pointed out that most aetiological hypotheses incorporate several factors which are described as being either causative or accessory in the production of senile entropion. The more commonly quoted factors are listed and examined in relation to the results of our investigations. A minority are supported to some degree but the majority are shown to be false concepts. The surgical implications of our findings are discussed.

It is hoped that this work will stimulate further research and that it will contribute towards the final elucidation of the aetiology of the condition, or group of conditions, we now call "senile entropion". When this has been achieved the final evaluation of the multiple corrective surgical procedures will be possible. In this light a plea is made for an end to the era of "surgical attack and aetiological speculation".

Our thanks are due to Mr. A. D. Mackenzie for his assistance with the clinical investigations, to Mr. R. L. Neave, medical artist, Manchester University, to Miss Kathleen Hughes, who prepared the histological sections, and to the Departments of Medical Illustration and Radiology at the Manchester Royal Infirmary. 


\section{REFERENCES}

Adler, F. H. (1947). “Gifford's Textbook of Ophthalmology, 4th ed., p. 159. Saunders, Philadelphia. ButLer, J. B. V. (1948). Arch. Ophthal. (Chicago), 40, 665.

Duke-Elder, S. (1952). “Text-book of Ophthalmology", vol. 5, p. 5184, Kimpton, London.

FFooks, O. O. F. (1961). Brit. J. Ophthal., 45, 130.

Foulds, W. S. (1961). Ibid., 45, 678.

Fox, S. A. (1951). A.M.A. Arch. Ophthal., 46, 424.

Fuchs, E. (1917). “Textbook of Ophthalmology" (trans. A. Duane), 5th ed., p. 678. Lippincott, Philadelphia.

JONES, L. T. (1960). Amer. J. Ophthal., 49, 29.

KeTteSY, A. (1948). ' Brit. J. Ophthal., 32, 311.

Kirby, D. B. (1953). Amer. J. Ophthal., 36, 1372.

LEBENSOHN, J. E. (1953). Ibid., 36, 504.

MCFARLANE, D. C. (1956). Ibid., 41, 657.

MeEK, R. E. (1940). Arch. Ophthal. (Chicago), $24,547$.

SchimeK, R. A. (1957). Amer. J. Ophthal., 43, 245.

Stallard, H. B. (1958). " "Eye Surgery.", 3rd ed., p. 186. Wright, Bristol.

Streatfield, J. F. (1858). Roy. Lond. Hosp. Rep., 1, 121.

SzlazaK, J. P. (1957). Amer. J. Ophthal., 43, 617.

Weeks, J. E. (1900). Trans. Amer. ophthal. Soc., 9, 18.

WolfF, E. (1958). "The Anatomy of the Eye and Orbit", 4th ed., p. 161. Lewis, London. 